\title{
Agent Affecting Cardiovascular System
}

National Cancer Institute

\section{Source}

National Cancer Institute. Agent Affecting Cardiovascular System. NCI Thesaurus. Code C78274

An agent that exerts a physiologic effect on any anatomic entity that is part of the cardiovascular system, including the heart and blood vessels. 